\title{
Addictovigilance : le défi pharmacologique de l'évaluation et de la prévention des substances à risque
}

Voilà désormais 25 ans que la France a mis en place un système organisé et opérationnel d'évaluation du potentiel d'abus et de dépendance des substances psychoactives, quels que soient leur origine et leur statut (substances illicites, médicaments, nouvelles substances de synthèse). Au fil des années, ce système, bâti autour de l'activité des Centres d'Évaluation et d'Information sur la Pharmacodépendance, est devenu d'addictovigilance. Encore une vigilance, direz-vous ! Certes, mais celle-ci est particulièrement d'importance, même si elle reste malheureusement trop méconnue et considérée comme un sous-ensemble de la pharmacovigilance classique. En effet, le domaine de l'abus et de l'addiction nécessite des méthodes d'exploration spécifiques et innovantes, sous peine de passer à côté de l'essentiel.

Ce numéro de Thérapie souligne l'originalité de ce domaine, et son nécessaire ancrage dans la culture pharmacologique, de la cible pharmacologique à la population générale, jusqu'à la société. Les différents points et les exemples abordés dans ce numéro illustrent l'importance d'une approche pharmacologique globale et intégrée pour l'évaluation du potentiel dépendogène d'une substance : considérant une cible d'action spécifique interférant avec l'activité du système nerveux central, cette boucle continue passe par la vérification expérimentale dans un modèle animal adapté, se poursuit par l'expérimentation clinique chez l'homme, puis à l'échelle de la population dans une approche pharmacoépidémiologique. Cette dernière approche permet l'identification de signaux, dont la pertinence et la signification clinique doit être explorée au regard des données pharmacologiques expérimentales et cliniques.

Les principaux outils de détection et d'évaluation des différentes substances à potentiel d'abus et de dépendance développés en France sont décrits, et remis en perspective pour présenter les moyens mis en œuvre pour identifier un signal en addictovigilance en France. La présentation des méthodes et des modèles animaux utilisés en neurobiologie illustre la nécessité de la prise en compte des résultats obtenus dans la compréhension, l'anticipation ou la confirmation de comportements d'abus et/ou de dépendance observés en addictovigilance. Afin de compléter ces éléments, les différents outils pharmacoépidémiologiques disponibles en Europe pour explorer le phénomène d'abus et de dépendance aux médicaments sont présentés. Cette revue de la littérature permet de mettre en évidence l'hétérogénéité des sources d'informations disponibles et de pressentir la marge d'amélioration des moyens d'évaluation à l'échelle européenne.

Enfin la dernière partie du numéro aborde, à partir d'exemples précis - nouvelles drogues de synthèse, méthylphénidate, méthadone, tramadol - les enjeux de l'addictovigilance pour la sécurité sanitaire des populations. 